$\mathbb{T}$ periodica polytechnica

Civil Engineering

$52 / 1(2008) 15,22$

doi: 10.3311/pp.ci.2008-1.02

web: http://www.pp.bme.hu/ci

(c) Periodica Polytechnica 2008

RESEARCH ARTICLE

\section{A probabilistic approach for a T-stub ultimate strength assessment using response-surface approximation}

\author{
František Kurčik / Abdelhamid Bouchaïr / Josef Vičan
}

Received 2007-12-05

\begin{abstract}
The main goal of the study is to examine and demonstrate the application of a developed probabilistic framework to the analysis of a chosen general type of steel connection component in order to obtain the basic characteristics of its mechanical behaviour with regard to the chosen type of the input geometry and material properties. Accordingly, as a typical representative of the component method proposed by the Eurocode the equivalent T-stub was chosen as a subject of the study. This type of structural element has the capability to address the behaviour of several parts of the connection: column flange in bending, end plate in bending and flange cleat in bending. A method is proposed to determine the probability density function of the ultimate strength using a response-surface approach coupled with FEM applied to a stochastic structural model. The results of a study were processed by means of sensitivity analysis to determine the importance level of the input variables.
\end{abstract}

\section{Keywords}

steel connection . T-stub ultimate strength $\cdot$ finite element model $\cdot$ response-surface method $\cdot$ stochastic model $\cdot$ Monte Carlo simulation $\cdot$ sensitivity analysis

\section{Acknowledgement}

This work was supported by the Slovak Research and Development Agency under the bilateral cooperation SK-Fr01706 and the contract number APVV20-010005 and by thr French Ministry of Foreign Affairs in the frame of the bilateral cooperation PHC STEFANEK 11251 VM 2006/2007.

\section{František Kurčik}

Department of Structures and Bridges, Faculty of Civil Engineering, University of Žilina, Komenského 52, 01026 Žilina, Slovak Republic

e-mail:kurcik@fstav.utc.sk

\section{Abdelhamid Bouchaïr}

Blaise Pascal University, CUST (PolyTech'Clermont-Ferrand), Civil Engineering Laboratory (LGC),, Rue des Meuniers, B.P. 206, F-63174 Aubière cedex e-mail: bouchair@cust.univ-bpclermont.fr

\section{Josef Vičan}

Department of Structures and Bridges, Faculty of Civil Engineering, University of Žilina, Komenského 52, 01026 Žilina, Slovak Republic e-mail: vican@fstav.utc.sk

\section{Introduction}

The main questions when we are dealing with the behaviour and functionality of basic structural components such as the steel connections consist of the identification and the qualitative evaluation of the critical parameters which can influence their resulting resistance, stiffness or deformability. These factors are crucial for the cost effective application of such types of elements considering their design and further exploitation within the structures. In general practice a large number of steel structures are commonly using the bolted connections as simple or moment resisting joints between structural members. Their popularity can be attributed mainly to the simplicity and economy attributed with design, fabrication and utilization in wide area. In the past, almost every application of structural design for steel frames has been considering a rigid or a pinned connection behaviour regardless of their real response. This was due to the possibility of important simplification in design process and the lack of simplified methods for the prediction of the required connection properties. However a significant gain can be achieved by incorporating the finite stiffness of connections in the design model based on the concept of the semi-rigid joints. Their mechanical behaviour described by the stiffness, the resistance and the rotational capacity is generally nonlinear because of the contact zone evolution, the plasticity of materials, the large displacement, etc. It can be described by an empirical approach based on tests, by a sophisticated numerical calculation or by an analytical method [1, 2]. The last one has been proved as most appropriate and it is generally known as the component method. This design philosophy proposed by the modern structural codes provides greater freedom in design because the properties of the connections are treated as variables and are resulting into their more economic application. The method itself consists of dividing the connection into several components each one modelled as a spring element. The Annex J [3] and the part 1.8 [4] of Eurocode 3 proposes expressions to quantify the contribution of each component and rules to combine them. One of the most significant components is the so called T-stub. Considering the current research tasks the main goal is to improve the knowlegde about the mechanical behaviour of the chosen type of the con- 
nection component in order to contribute to the coherent design rules for structural connections.

This can be achieved by the several different methods. One typical option is to perform the experimental research as it was already done throughout the recent years, but despite the extensive effort and lot of advances, many questions are still unanswered [5]. Another possibility is to employ nonlinear FEM modelling employing advanced numerical models [6, 7]. This approach is rather applicable because of significant reduction of expenses and time related with experimental investigation in laboratory. Another advantage is the possibility to perform parametric studies which can give information about importance of various structural elements from which a T-stub is a part. On behalf of this aim a modelling using probabilistic framework often takes place [8].

According to this statement, in this study the approach using nonlinear finite stochastic model was created to perform the probabilistic analysis of the equivalent T-stub to quantify the effects of some parameters variability on its resulting behaviour and its ultimate bearing capacity. A method is proposed to determine the probability density function of the ultimate bearing capacity of the T-stub using a response-surface approach. Finally, the statistical model for the output quantity is presented, which can be used in the form of a simple mathematical formula for the parametric studies and for the description of the overall behaviour of the T-stub, which is relatively complex in terms of the normative approach and the mechanical behaviour. The complementary goal is to carry out the sensitivity factors wich are determining the importance level of input variables on the response of this investigated structural component.

\section{Connection modelling}

Bolted connections are used widely in steel frames as simple or moment resistant connections between steel members. Their characteristics must be taken into consideration to accurately predict the behaviour of the whole structure. In almost every practical case of structural design for steel frames the real response of the connections is of little interest since they are considered having ideally rigid or pinned behaviour. This approach greatly simplifies the design process, mainly not by the consequent reduction of the design variables but by the impact these variables have in the analysis results. However, a significant gain in the structural weight could be achieved by incorporating the finite stiffness of the connections in the design model, and performing an optimization technique. The internal forces could be then more advantageously distributed among structural members, which can lead to more efficient material use.

Furthermore, the cost of the so-called semi-rigid connections is generally lower compared to that of rigid ones, providing an additional profit. The increased computational cost and mainly the lack of simplified methods for the prediction of required connection properties, such as initial stiffness, strength and rotational capacity, prevents the adoption of semi-rigid connections in every-day design practice. The normative approach [3, 4] proposes an analytical procedure for this purpose, but it is rather complicated for practical use without automatic calculation,

This paper describes the properties and the performance of a finite element model for the simulation of a simple T-stub steel connection. It is a first step before the extension to more complex connections, such as bolted end-plate ones. A valid numerical model is an essential tool in this context, since a large amount of parametric analyses is required in order to simplify the analytical methods, covering both static and dynamic problems, to become a reality. This treatment of steel connections is representative of the continuously increasing use of advanced numerical analysis as a tool for practical design of engineering structures in general and steel structures in particular. The recent progress in hardware and non-linear numerical algorithms is expected to affect design codes and design methodologies in the coming years. Current codes do recognize this progress by allowing structures to exhibit non-linear behaviour, both from a material and a geometry point of view. Treatment of these nonlinearities is, however, still performed in a rather crude way, and is mostly based on linear elastic analyses followed by several checks for dimensioning, making use of approximations, such as effective buckling lengths for global buckling, classification into slenderness categories for local buckling, seismic behaviour factors for inelastic response during extreme seismic events, etc. This is at the expense of accuracy and reliability, and intensifies significantly the dimensioning and checking process. The progress in computational mechanics with emphasis on reliability and efficiency is expected to support the use of more rigorous analysis procedures that can take into account all these sources of non-linearity, predict the pertinent failure modes, and thus increase accuracy on one hand, and reduce the effort involved in checking on the other.

As it was noted above, one of the most important concepts for the component method is the equivalent T-stub idealization of the tension zone of a connection. Therefore, this tension zone is substituted by a T-stub section of appropriate effective length (see Fig. 1).
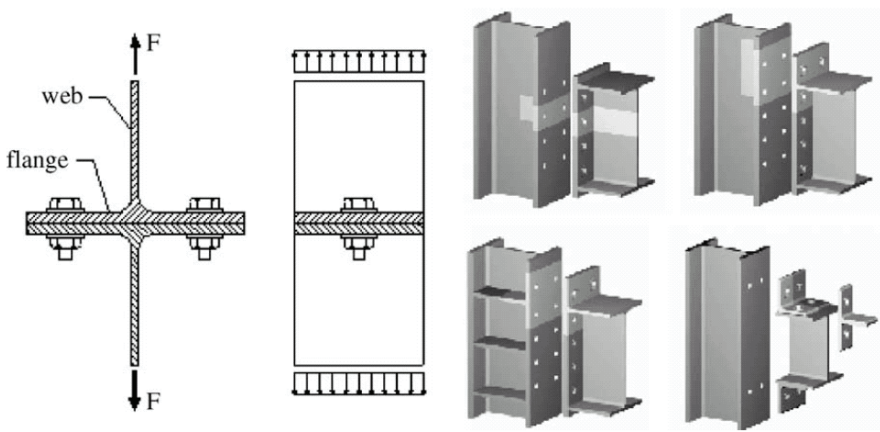

Fig. 1. T-stub geometry and positioning of T-stubs in bolted beam-tocolumn connections

In spite of being much simpler than a usual bolted conection it has been proven to adequately model parts of the connection 
under tension. It is composed of two T-shape sections connected by means of one or more pair of bolts. This connection may fail according to one of the three known failure mechanisms shown in Fig. 2. The first one occurs with the development of four plastic hinges in the flange plates located at the bolt holes and at the flange to web fittings. The second one occurs with the development of two plastic hinges in the flange plates positioned at the flange to web fittings and with axial failure of the bolts. The third failure mechanism occurs with axial failure of the bolts accompanied by full separation of the flange plates. This simple connection is not directly used in frame designs. It is, however, one of the components of bolted beam-to-column joints, determining their stiffness, strength and rotational capacity.
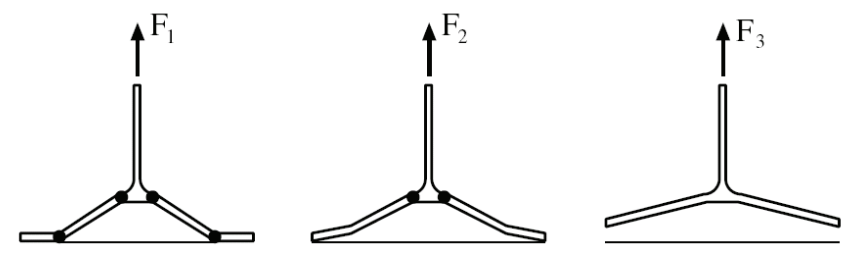

- Plastic hinge

Fig. 2. Failure mechanisms of T-stub connections

In Fig. 1 the positioning of T-stubs in various classes of bolted beam-to-column connections is also presented.

\section{Stochastic structural model}

A general concept for the creation of a finite element model is presented. Then it is applied for the modelling of an existing T-stub for which the experimental results are available. An early decision to be made in almost every numerical simulation concerns the model space dimension. For a T-stub, 2D and 3D models are possible and have been implemented in the literature [9. 10]. The 2D model has been used as an acceptable compromize between accuracy and computational cost. For this study the $2 \mathrm{D}$ model is chosen. A substantial reduction of the model size can be achieved by exploiting the symmetries of the problem. In a T-stub connection two planes of symmetry can be defined. Thus, only one quarter of the whole connection needs to be modelled, as shown by the highlighted part of Fig. 3 Thus, the variability is considered as symmetrical for the T-stub. The reduced model needs to be completed by suitable boundary conditions in the symmetry planes, constraining normal to plane displacements and in-plane rotations.

The stochastic structural model required for probability analysis was created using multipurpose finite element code ANSYS [11] employing SHELL93 finite elements permitting to perform nonlinear solution for problems of large deformations analysis. It is capable of reproducing contact and friction phenomena and offers sufficient handling options for material and geometric nonlinearities. The mesh on the Fig. 3 consisted of 154 elements and 553 nodes with allowed quadrilateral meshing procedure for element shapes, which led to the solution of about 3500 equations.

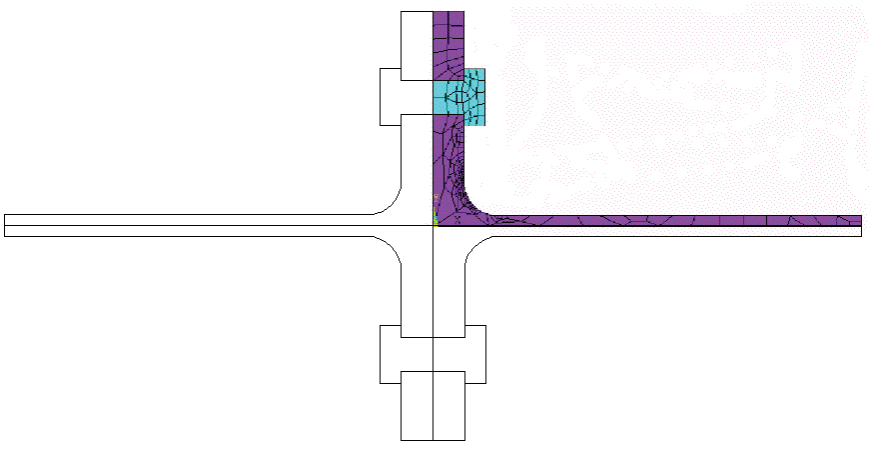

Fig. 3. T-stub mesh and quarter symmetry

For the simulation of the various contact phenomena, efficient general algorithms have been published [12]. In our study the CONTA174 and TARGE170 elements were used in order to simulate the effects of surface-to-surface contact zone between flange plates and between bolt and flange plate. A zero friction coefficient was used in the model [6]. The Augmented Lagrange Method with contact detection on gauss points including initial penetration and stiffness updating in each load step was used. An isotropic type material was chosen with bilinear stress-strain law and isotropic hardening rule. The well suited von Mises yield criterion for steel elements was chosen. To simulate the variance of the material properties in the T-stub, different materials were assigned for flange, web plate and bolt. Thus, the material strength characteristics are considered as random variable, i.e. steel yield stress and strength which are different for the flange, web and bolts. The Young's modulus of elasticity $\mathrm{E}=$ $210 \mathrm{GPa}$ and the Poisson's coefficient 0,3 are considered as constant due to their low variability. Incorporating of random input variables for purposes of the probabilistic analysis is possible by modification of the input program code using the APDL language (Ansys Parametric Design Language) and consequently to utilize them within the integrated PDS module (Probabilistic Design System), which is permitting direct connection between the FEM database and the chosen probability analysis algorithm [11]. Further description of this procedure and related statistical models of selected random input variables will be given later. Dimensions and application of this FEM model is explained in the following part.

\subsection{Model validation}

In order to validate the performance of the numerical model, experimental data from the literature were used. Bursi and Jaspart described a series of physical T-stub tests [13]. These tests were used for the creation of a T-stub numerical benchmark [14] and have been used by many investigators, thus their geometry and material properties are well documented. In these tests various different available specimen configurations have been tested to evaluate the effect of different geometric and strength parameters.

For our study, a specimen named T1 have been selected. It is characterized by a relatively weak flange plate and, therefore, 
it was carried out that the first failure mechanism is critical for this type of configuration (see Fig. 2). Geometrical properties for the chosen type of specimen are shown in Fig. 4 and detailed description considering material properties for flange, web and bolt can be found in literature [13].

Comparison between the experimental measurements and the results of the numerical analysis was performed to verify the accuracy and the validity of the finite element model. For this objective the Arc-Length method was used for the simulations of the ultimate strength of T-stub. This method is suitable for nonlinear static equilibrium solutions of unstable problems. Application involves the tracing of a complex path in the loaddisplacement response into different response regimes. Mathematically, the Arc-Length method can be viewed as the trace of a single equilibrium curve in a space spanned by the nodal displacement variables and the total load factor. In our study the total load factor was a single scalar parameter defined by the time step of value equal to $1 / 10$. Simulations of the ultimate strength were carried out while searching for the limit point using explicit spherical iterations with the following Arc-Length algorithm :

$$
\left[K_{i}^{T}\right]\left\{\Delta u_{i}\right\}=\lambda\left\{F^{a}\right\}\left\{F_{i}^{n r}\right\} \text { with }:-1.0 \leq \lambda \leq 1.0
$$

where $\left[K_{i}^{T}\right]$ is the Jacobian tangent stiffness matrix, $\left\{\Delta u_{i}\right\}$ is the displacement vector, $\left\{F^{a}\right\}$ is the vector of applied loads, $\left\{F_{i}^{n r}\right\}$ is the resisting loads vector and $\lambda$ is the proportional loading factor. The limit point can be found by performing iterations until the tangent stiffness is zero. The physical interpretation of this point can be assigned to the collapse behaviour of the T-stub.

On Fig. 5 the comparison between the experimental and the numerical force-displacement curves of specimen T1 is shown, where (F) is the total applied force (shown in Fig. 1) and (d) the corresponding displacement. The curve match is satisfactory for the elastic region and part of the plastic region. Both initial stiffness and strength are predicted accurately. However, we can notice that the $2 \mathrm{D}$ model shows rather stiffer behaviour. The deformed geometry at the failure stage shown in Fig. 6 indicates the occurrence of the first failure mechanism according to the EC3 and confirms the good performance of the model and its ability to reproduce the kinematics of bolt-plate interaction. In the same figure the von Mises stress and the axial stress in a plane perpendicular to the web of the T-stub are depicted. Two plasticity zones are formed in the flange plate, along the bolt line and in the toe of the fillet, while the behaviour of the bolt is mainly tension and bending with partial plastification of its shank. These remarks are validated by the experimental results.

Some preliminary studies which were already carried out in this area [9] showed that the relevant geometrical variables considering the ultimate strength of the T-stub and its overall performance are the thickness of flange $\left(\mathrm{t}_{f}\right)$, the flange width (b), the diameter of the bolt (d) and the size of the bolt head (e) and (k). Relevance of the individual random variables for geometry is shown in Fig. 4. It was considered that all these variables had a normal distribution and the upper and lower limits of the parameters were 105 and $95 \%$ of the nominal value.

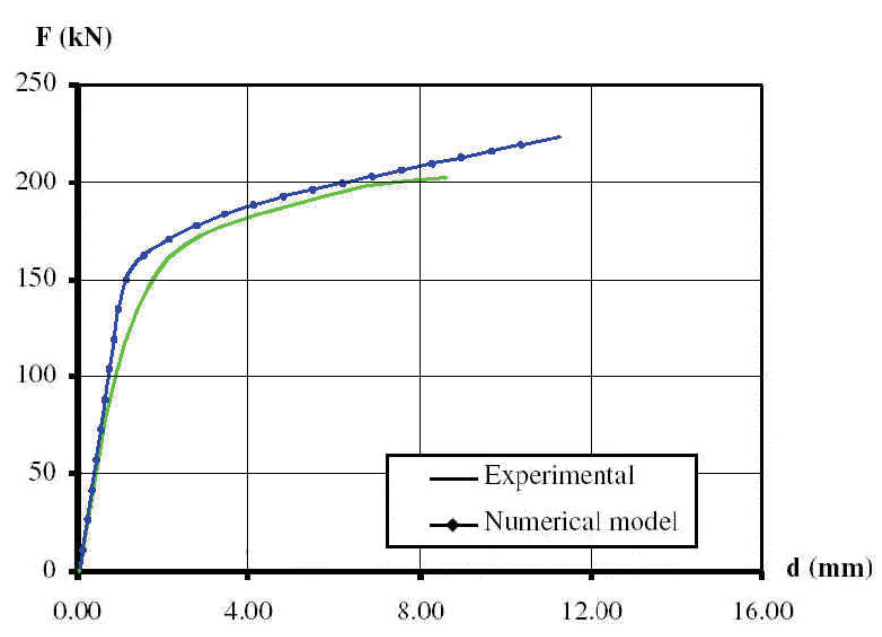

Fig. 5. Load-displacement curve for the model $\mathrm{T} 1$ and comparison with the experimental results

Afterwards, the scatter of these variables was considered taking into account the dimensions and the tolerances given by the standards (EN 10034 [15] and ISO 4014 [16]). For the rolled section an upper and lower value of the tolerances are given. The scatter of the variables was simulated by a normal distribution, $\mathrm{N}\left(\mu, \sigma^{2}\right)$, where the mean, $\mu$, and the standard deviation, $\sigma$, are given by: $\mu=(U+L) / 2$ and $\sigma=(U-L) /(2 \times 3.09)$, where $U$ is the upper value and $\mathrm{L}$ the lower. For some of the bolt dimensions only the nominal values and a minimal value were given in the standards. For this a normal distribution was also admitted, with the upper tolerance equal to the lower tolerance. The other values were calculated as before. Table 1 summarizes the standard dimensions adopted for the bolts and the rolled section. Taking into account the values defined in Table 1 , the parameters which describe the distributions of the random input variables are evaluated according to the presented expressions.

For the description of the random input variables entering calculation, the truncated normal Gaussian distribution function was used to override possible numerical issues due to the well known tail problem in numerical simulations using extreme values. The presented approach was used in the probabilistic simulation framework. It involves consecutive repeating of calculations with randomly generated subsets of the input variables and the evaluation of the response in the form of the so called limit surface. There is a large amount of methods which could be possibly used, but as it was previously stated, the use of the response-surface methodology coupled with FEM code seems to be one of the most efficient approaches although it is not yet widely used.

\section{Response-surface method for ultimate strength of the T-stub}

In many practical applications and in particular, in the prediction of the maximum load-carrying capacity of structures in the 


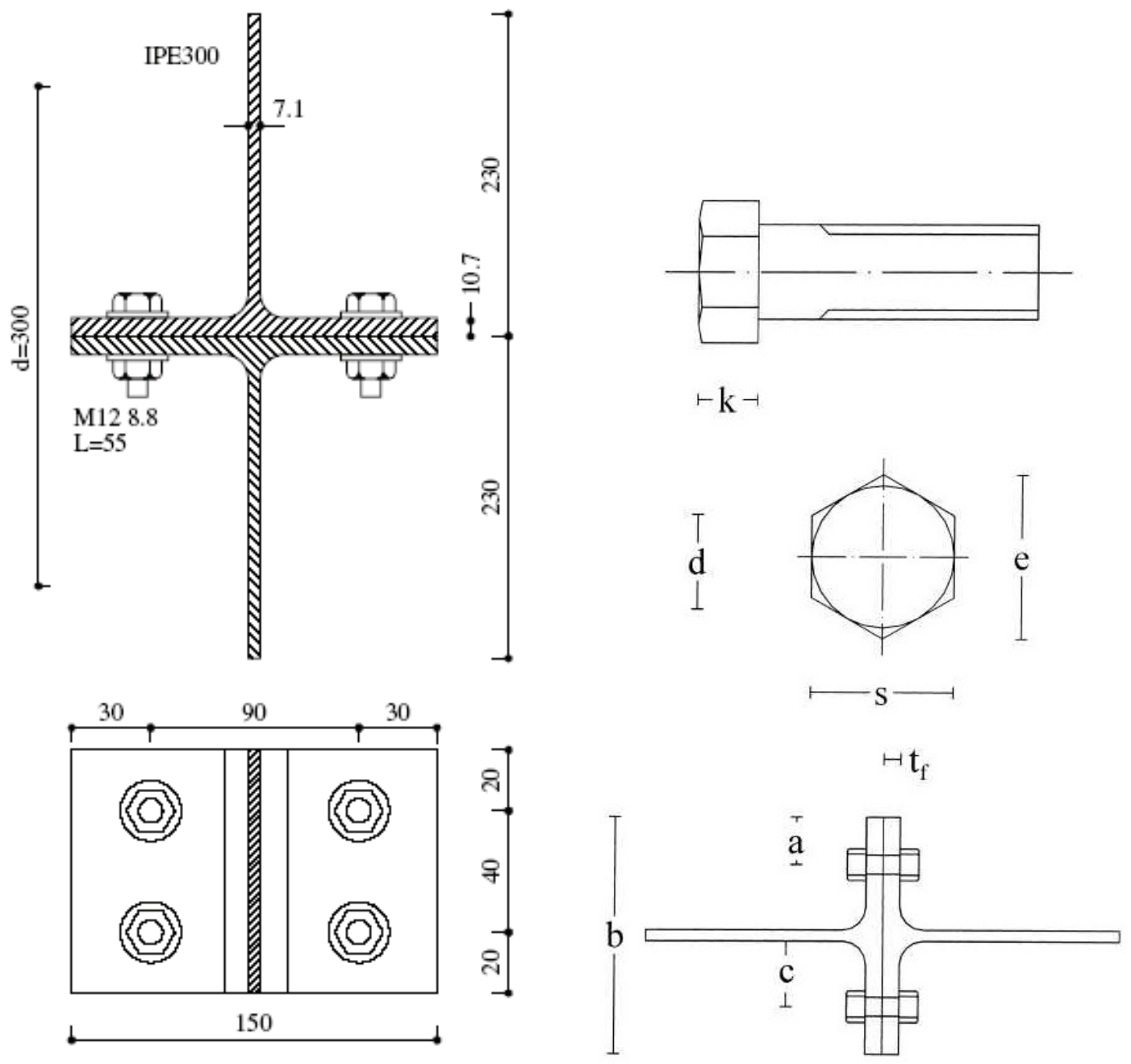

Fig. 4. Geometrical properties of $\mathrm{T} 1$ with bolt and profile geometries
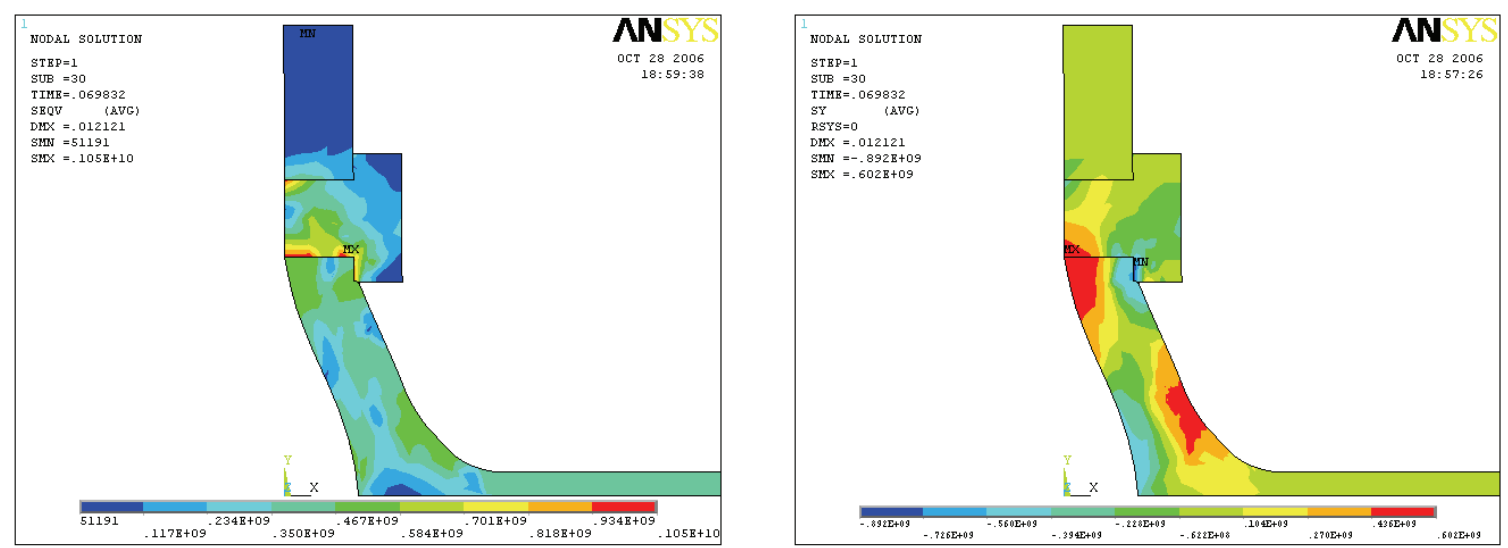

Fig. 6. Von Mises stresses at failure stage of $\mathrm{T} 1$ model (left) and stresses in the $\mathrm{Y}$ axis (right)

Tab. 1. Statistical models of the random input variables

\begin{tabular}{llllllll}
\hline Dimension $(\mathrm{mm})$ & Minimum & Nominal & Maximum & $\mu$ & $\sigma$ & $\sigma$ & $\mu(\%)$ \\
\hline $\mathrm{t}_{f}$ & 9.20 & 10.70 & 13.20 & 11.20 & 0.3236 & 2.89 \\
$\mathrm{~b}$ & 148.0 & 150.00 & 154.00 & 151.00 & 0.4854 & 0.32 \\
$\mathrm{~d}$ & 11.73 & 12.00 & 12.00 & 11.87 & 0.0218 & 0.18 \\
$\mathrm{e}$ & 20.47 & 20.78 & 21.10 & 20.78 & 0.0504 & 0.24 \\
$\mathrm{k}$ & 7.32 & 7.50 & 7.68 & 7.50 & 0.0291 & 0.39 \\
\hline
\end{tabular}


elasto-plastic range, the analytical expression for the response of a structure often does not exist. A possible alternative is to use approximate functions formulated on a semi-empirical basis, which are normally derived after some reduction of the number of random variables on which the structural ultimate strength may depend. Consequently, this introduces additional model uncertainties in the analysis concerning the real behaviour of a structure. However, the level of uncertainty introduced in the model depends on the approach adopted. Some authors simply perform a regression analysis on the available data and this is a bound to increase the uncertainty and to limit the range of applicability of the formula to the range of the available data. The limitation of these approaches is that the available data do not always represent well the full range of variation of the governing parameters. A response-surface methodology combined with the numerical evaluation of the ultimate strength can be an answer to this problem [8]. This approach can have different strategies for covering the relevant range of variation of the parameters. Furthermore, combination with a non-linear finite element analysis ensures that all details such as initial imperfections are taken into account in the prediction of the value for the selected output variable.

To describe the main features of the method adopted here, it is necessary to determine an approximation to the ultimate capacity $\Phi$ of the structure, which is a function of $k$ independent variables, $X_{1}, X_{2}, \ldots, X_{k}$. This means to determine the unknown coefficients $a_{i}$ in the following expression:

$$
\phi=a_{0}+a_{1} x_{1}+a_{2} x_{2}+a_{3} x_{3}+\ldots+a_{k} x_{k}
$$

When $n$ calculations have been performed, with $k$ variables defining the input at each run, in the present case by a nonlinear finite element code, then the following set of equations is obtained by:

$$
\phi_{i}=a_{0}+a_{1} x_{1 i}+a_{2} x_{2 i}+a_{3} x_{3 i}+\ldots+a_{k} x_{k i}, i=1, \ldots, n,
$$

where $\phi_{i}$ is the ultimate capacity that resulted in the i-th case, which is calculated with the sample of the values associated with the variables $X_{i j}$ (for $j=1,2, \ldots, k$ ). This sample can be determined by techniques of experimental design, which provides the best combination of parameters to cover the range of variation of the variables, or it can just be obtained by random sampling each variable.

The equations can also be written as :

$$
\Phi=X a
$$

where

$$
\Phi^{T}=\left[\phi_{1}, \phi_{2}, \phi_{3} \ldots, \phi_{n}\right]
$$

is the vector of the structural load-carrying capacities obtained from the finite element calculations,

$$
a^{T}=\left[a_{1}, a_{2}, a_{3}, \ldots, a_{n}\right]
$$

is the vector of the unknown coefficients, and

$$
X=\left[\begin{array}{llll}
1 & x_{11} & x_{21} & x_{31} \ldots x_{k 1} \\
1 & x_{12} & x_{22} & x_{32} \ldots x_{k 1} \\
\ldots & \ldots & \ldots & \ldots \\
1 & x_{1 n} & x_{2 n} & x_{3 n} \ldots x_{k n}
\end{array}\right],
$$

is the matrix of the values adopted in the calculations for the basic variables considered.

Multiplying (3) by $X^{T}$ and assuming that $X^{T} X$ is nonsingular, the following estimation of the vector of the values of the unknown coefficients can be obtained

$$
a^{T}=\left(X^{T} X\right)^{-1} X^{T} \Phi
$$

which is the last-squares estimator of the vector $a$

Having the vector of the coefficients $a_{i}$, it is then possible to have an approximation to the response-surface that describes the ultimate strength of the T-stub, as given by Eq. (1). This expression can be used to construct a limit state function by completing it with the description of the loading.

The approximation around mean values of the random variables can also serve as a useful tool when evaluating the mean value and the standard deviation of the T-stub ultimate strength. If the approximation $\Phi$ is a linear function of the kind of Eq. (1) with $k$ correlated random variables with their mean values $\mu_{1}$, $\mu_{2}, \ldots, \mu_{k}$ and standard deviations $\sigma_{1}, \sigma_{2, \ldots,} \sigma_{k}$ then its mean and standard deviation would be:

$$
\begin{gathered}
\mu_{\Phi}=a_{0}+\sum_{i}^{k} a_{i} \mu_{i} \\
\sigma_{\phi}=\left(\sum_{i}^{k} a_{i}^{2} \sigma_{i}^{2}+\sum_{i}^{k} \sum_{j}^{k} \rho_{i j} a_{i} a_{j} \sigma_{i} \sigma_{j}\right)^{1 / 2},
\end{gathered}
$$

where $\rho_{i j}$ are the correlation coefficients between the variables $X_{i}$ and $X_{j}$.

\section{Numerical analysis}

The results of the numerical simulations for the ultimate strength are presented in the form of explicit approximation function, which defines the stochastic response of the structure on the random input variables. As it was already introduced, we are accepting the assumption, that the influence of the random input variables on the random output parameters can be approximated by a mathematical function. Hence, the response-surface method locates the sample points in the space of random input variables such that an appropriate approximation function can be found most efficiently. According to these statements, the analysis consisted of two steps.

Firstly, it was required to perform the simulation loops to calculate the values of the random output parameters that correspond to the sample points in the space of random input variables. This was made using the Central Composite Design (CCD) algorithm for 27 loops of about 30 minutes per each. 
Secondly, a regression analysis using the least squares methods was performed to derive the terms and the coefficients of the approximation function. The resulting output variable is the ultimate strength for the first failure mode of the T-stub. The basic idea is that once the coefficients of a suitable approximation function are known, then we can directly use the approximation function instead of looping through the finite element model. The values of the ultimate strength of the T-stub were then calculated using Monte Carlo (MC) simulation on the responsesurface approximation. It is noted, that the number of MC simulation is not essential for the accuracy of solution, because it depends mainly on number of CCD loops and quality of sampling procedure. On the following Fig. 7 the output histogram for ultimate strength and related limit surface are depicted.

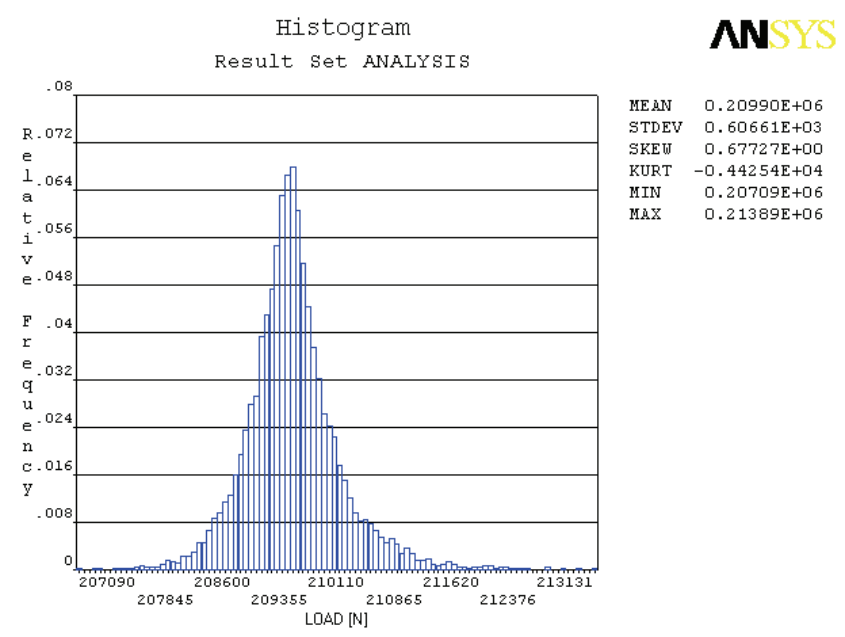

Fig. 7. Histogram of ultimate strength of the T-stub obtained by means of MC simulations

\section{Sensitivity analysis}

As a complement to the reliability analysis it is possible to evaluate the sensitivity factors. These factors are defined by the square of the coordinates of the unit vector which sum up to 1 . Generally written in percentage, these factors allow ranking of the basic random variables according to their importance in the reliability analysis. In this study their values were calculated using Spearman rank order coefficients [11] and their values are shown on Fig. 8. The sample correlation with cubic trendline between resulting ultimate strength and one of the chosen input geometry variable of the flange width (b) is also presented.

From the figures we can see that the importance level of the flange width (b), the thickness of the flange $\left(\mathrm{t}_{f}\right)$ and the size of the bolt head $(\mathrm{k})$ is higher, while the importance of other characteristics of the geometry like diameter of the bolt (d) is remaining to be more or less complementary. These results were confirmed by the matrix of the rank order correlation coefficient evaluation which showed the highest significance of $\mathrm{t}_{f}, \mathrm{~b}$ and $\mathrm{k}$ for the resulting value of ultimate strength.

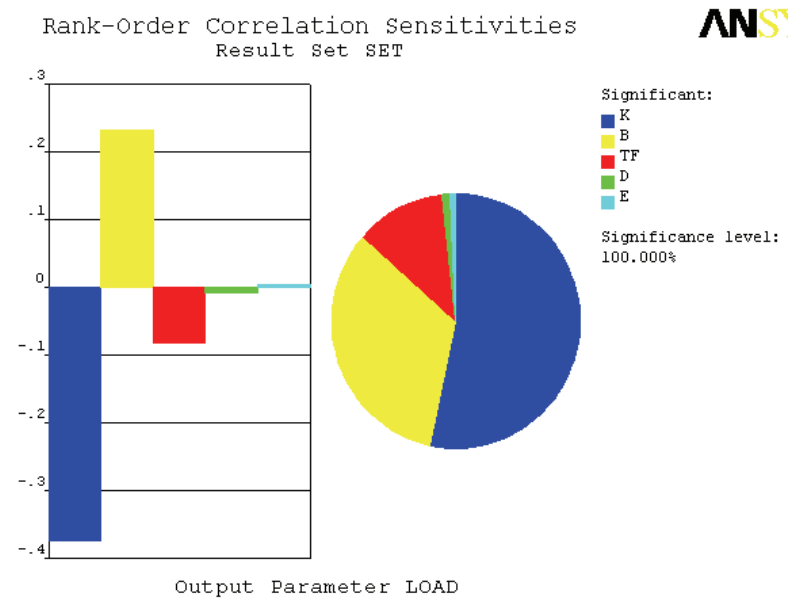

Fig. 8. Sensitivity factors for random input variables

\section{Conclusions}

A T-stub stochastic finite element model was developed. Its performance in reproducing the actual load-displacement curve was tested with experimental data and proved satisfactory with a chosen configuration. A method was proposed to determine the probability density function of the ultimate strength using a response-surface approach. It was shown that this procedure can combine the accuracy of the FEM and the efficiency of the approximative analytical models and is versatile in terms of computation time and also in cases when the limit state of the structure does not exist in an explicit form. As a result of the performed probabilistic analysis, the importance of the geometrical T-stub properties for the resistance was verified in terms of sensitivity analysis. Presented methodology can be effectively applied to any other types of the steel structural elements which are commonly used in practice. The approach introduced in this study will be applied to other geometrical and material configurations of T-stubs, considering the steel plate and the bolt, which allow the development of the three recognized failure modes. After the complete validation of the mechanical and the probability approaches, the study will be extended to the case of the whole joint with the aim of the evaluation of the life cycle by considering for example the corrosion of the steel into various conditions of exposure.

\section{References}

1 Maggi YI, Goncalves RM, Leon RT, Ribeiro LFL, Parametric analysis of steel bolted end plate connections using finite element modelling, Journal of Constructional Steel Research 61 (2005), 689-708.

2 Bahaari MR, Sherbourne AN, Finite Element Prediction of End Plate Bolted Connection Behaviour : II- Analytic Formulation, Journal of Structural Engineering 123 (1997), 165-175. ASCE.

3 ENV-1993-1. J Eurocode 3: design of steel structures. Part 1.1: General rules and rules for buildings Annex J - joints in building frames, Brussells, 1994. European Committee for Standardisation (CEN).

4 Eurocode 3, Design of steel structures, part 1.8: Design of joints, 2005. CEN.

5 Bursi OS, Jaspart JP, Basic Issues in the Finite Element Formulation of 
Extended End Plate Connections, Computers and Structures 69 (1998), 361382.

6 Al-khatab Z, Analysis of the behaviour of steel joints strengthened by backing-plates, numerical approach and experimental calibration, Clermont 2 (France), 2003 (French). PhD thesis, Blaise Pascal University.

7 Al-khatab Z, Bouchaïr A, Analysis of T-stub strengthened by backingplates with regard to Eurocode 3, Journal of Constructional Steel Research 63 (2007), 1603-1615.

8 Sudret B, der Kiureghian A, Stochastic finite element method and reliability, Berkeley, 2000. University of California.

9 Neves LAC, Cruz PJS, Henriques AAR, Reliaility analysis of steel connection components based on FEM, Engineering Failure Analysis 8 (2001), 29-48.

10 Gantes CJ, Lemonis ME, Influence of equivalent bolt length in finite element modeling of T-stub steel connections, Computers and Structures 81 (2003), 595-604.

11 Reh S, Beley DJ, Mukherjee S, Eng Hui K, Probabilistic finite element analysis using ANSYS,Structural Safety, Elsevier, 2006.

12 Moro T, El Hami A, EI Moudni A, Reliability analysis of a mechanical contact between deformable solids, Probabilistic Engineering Mechanics 17 (2006), 227-232.

13 Bursi OS, Jaspart JP, Benchmarks for Finite Element Modelling of Bolted Steel Connections, Journal of Constructional Steel Research 43 (1997), 17 42.

$14 \ldots$ _ Calibration of a Finite Element Model for Isolated Bolted End-Plate Steel Connections, Journal of Constructional Steel Research 47 (1997), 225262.

15 EN 10034. Structural steel I and H sections - Tolerances on shape and dimensions, Brussells, 1993. European Committee for Standardisation (CEN).

16 ISO 4014. Hexagon head bolts - Product grades A and B., Brussells, 2000. European Committee for Standardisation (CEN). 